\title{
Comunicación
}

\section{Shock hipovolémico por ruptura aórtica en perro: un caso de espirocercosis}

\author{
Hypovolemic shock due to aortic rupture in dogs: a case of spirocercosis \\ Teresa Oviedo-Socarrás ${ }^{1,2}$, Rodrigo De Lavalle G. ${ }^{1 .}$
}

\section{Resumen}

Se presenta un caso de espirocercosis canina, que durante el proceso de migración de la larva culminó en ruptura de la pared de la aorta, con consecuente shock hipovolémico y muerte de un canino de raza Pointer de dos años.

Palabras clave: espirocercosis; shock; ruptura aórtica; hemorragia

\section{AbSTRACT}

It is reported a case of canine spirocercosis, which during the migration process of the larva culminated in rupture of the aortic wall, with consequent hypovolemic shock and death of a two-year-old Pointer dog.

Key words: spirocercosis; shock; aortic rupture; haemorrhage

\footnotetext{
${ }^{1}$ Facultad de Medicina, Veterinaria y Zootecnia, Universidad de Córdoba, Montería, Colombia
}

${ }^{2}$ E-mail: toviedo@correo.unicordoba.edu.co 


\section{INTRODUCCIÓN}

La hemorragia masiva es una emergencia médica con graves implicaciones para el paciente; la pérdida significativa y rápida de volumen sanguíneo circulante conduce en forma secuencial a inestabilidad hemodinámica, disminución de la perfusión, hipoxia tisular, daño orgánico irreversible y muerte del individuo. Este cuadro clínico es conocido como shock hipovolémico o shock hemorrágico y puede ser ocasionado por múltiples causas (Gutierrez et al., 2004), entre ellas la ruptura de la aorta (Mozzer y Lima, 2012).

La ruptura de la aorta es una patología grave y con desenlace generalmente fatal (Banning y Pillai, 1997), cuyas causas pueden atribuirse a hipertensión sistémica (Wey y Atkins, 2000), aterosclerosis, malformaciones congénitas o defectos heredables en los componentes del tejido conectivo de la pared del vaso (Biasato et al., 2018, Boulineau et al., 2005) y migración de larvas de parásitos a través de la pared (Joubert et al., 2005).

Aunque es un evento raro, la mayoría de los casos registrados de ruptura aórtica en perros domésticos y cánidos salvajes son atribuidos al parásito Spirocerca lupi ( $S$. lupi). Los signos clínicos típicos de la espirocercosis son regurgitación, vómitos y disnea. El ciclo de vida del parásito involucra huéspedes intermedios como el escarabajo coprófago y diversos huéspedes paraténicos. Una vez ingeridas, las larvas penetran a través de la mucosa gástrica del huésped, migrando a lo largo de las arterias hasta la aorta torácica; finalmente atraviesan la pared de la arteria hasta su localización final que es el esófago caudal. Aquí, el parásito vive dentro de nódulos constituidos principalmente por fibroblastos (van der Merwe et al., 2008).

El diagnóstico precoz de la infestación por $S$. lupi sigue siendo un desafío en la práctica médica veterinaria. A la fecha, se han utilizado diversos fármacos para el tratamiento y la profilaxis de la enfermedad; no obstante, ninguno ha sido eficaz para matar estadios adultos y larvales del parásito sin efectos secundarios del huésped (van der Merwe et al., 2008).

La espirocercosis puede originar lesiones que implican proliferación mesenquimal, como son la osteopatía hipertrófica, espondilitis y sarcoma. Kirberger et al. (2013) sugieren que el nematodo $S$. lupi secreta o induce directamente la secreción de factores humorales que estimulan las células mesenquimales originando así este tipo de lesión; sin embargo, el daño a la pared de la aorta por migración parasitaria y la consecuente formación de aneurismas es la lesión más comúnmente asociada a espirocercosis (Bailey, 1963; Ramachandran et al., 1984).

En el presente trabajo se describe un caso de shock hipovolémico en perro Pointer, derivado de ruptura aórtica por $S$. lupi.

\section{Caso Clínico}

Canino hembra de raza Pointer, de 2 años y $23 \mathrm{~kg}$ de peso, fue llevada por su propietario sin signos vitales a la sala de necropsias de la Facultad de Medicina Veterinaria y Zootecnia de la Universidad de Córdoba, Colombia. Se indicó que el animal tenía un plan de vacunación completo, no tenía antecedentes de padecimientos clínicos, ni procedimientos quirúrgicos o tratamientos farmacológicos recientes, y que presentó muerte súbita en horas de la madrugada.

Se recibe el cadáver frío, iniciando fase de rigor mortis, sin signos apreciables de autólisis y en buena condición corporal. Las mucosas oral, conjuntival, vaginal y rectal presentaron palidez extrema. Al hacer la incisión y desprendimiento de la piel se observó palidez del tejido subcutáneo y nódulos linfáticos explorables sin tumefacción. En la cavidad torácica se observó hemotórax severo, pulmones ligeramente colapsados y co- 

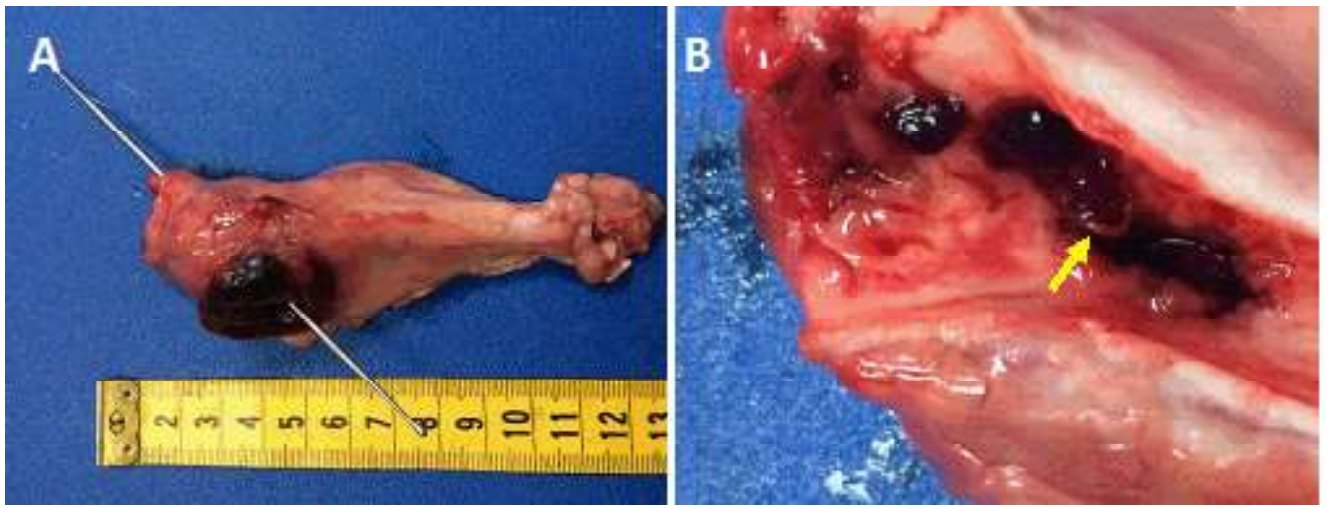

Figura 1. Ruptura de aorta en canino hembra de raza Pointer de 2 años. A. Disección de aorta donde se observa un hematoma de $3 \mathrm{~cm}$ en la pared con aparente perforación en el centro. B. Pared interna de la aorta con presencia de coágulo en el sitio de la ruptura y asociado a estructura parasitaria (flecha)
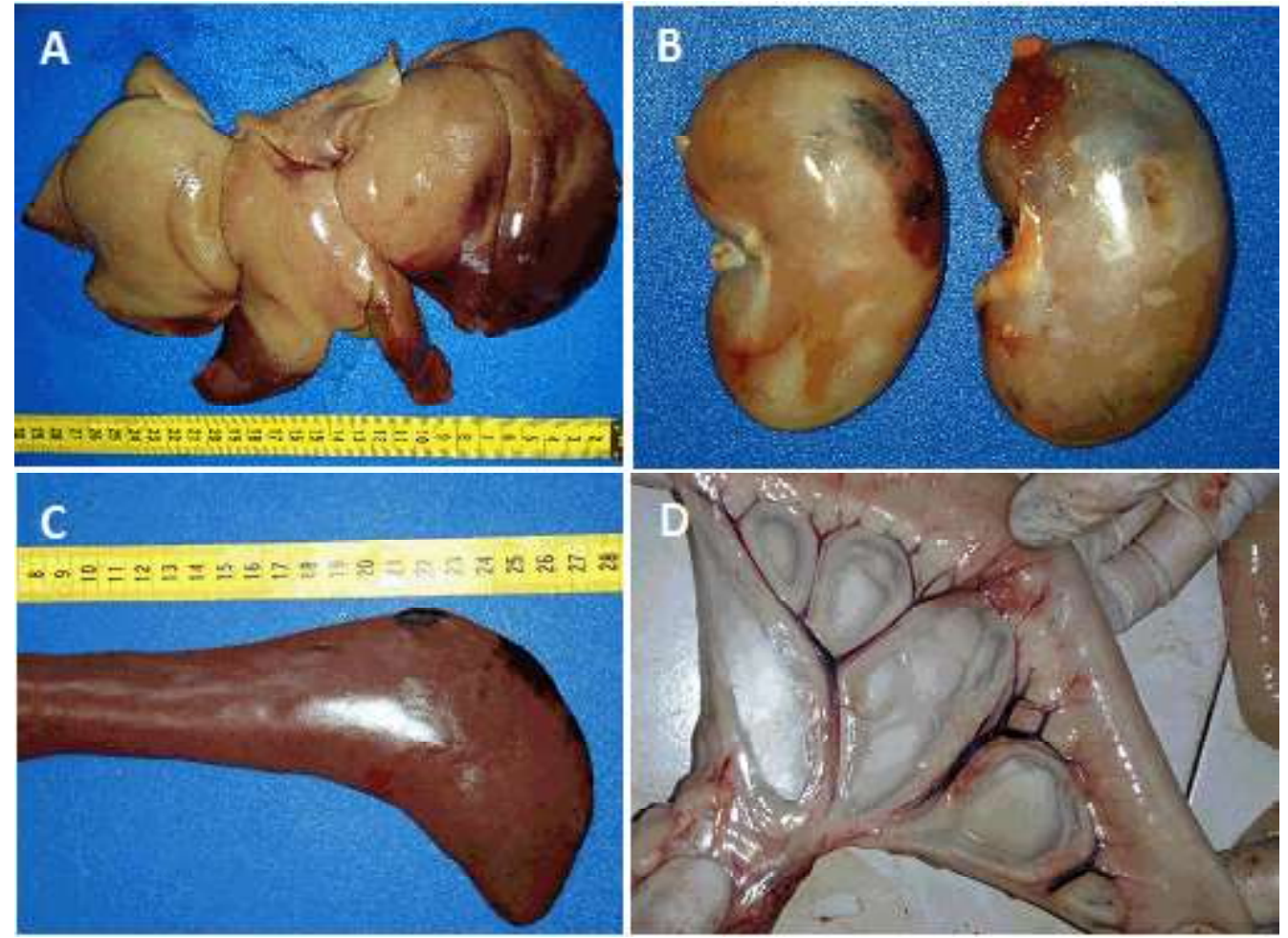

Figura 2. Hallazgos macroscópicos en canino hembra de raza Pointer de 2 años con muerte por ruptura aórtica. A. Palidez difusa severa del hígado. B, C y D. Áreas de infarto en riñones, bazo y presencia de coágulos en vasos mesentéricos 
razón con características de tamaño y forma normal. Al retiro de los coágulos sanguíneos de la cavidad torácica y de los órganos se observó que la pared de la arteria aorta, a unos $5 \mathrm{~cm}$ del hiato aórtico, presentaba un engrosamiento de forma redondeada de unos $3 \mathrm{~cm}$ de diámetro y color púrpura compatible con un hematoma (Figura 1A). En la disección del vaso se evidenció la pérdida de continuidad en la pared y la presencia de una estructura parasitaria compatible con larva de $S$. lupi envuelta por coágulos sanguíneos (Figura 1B). En la cavidad abdominal, se observó una palidez severa difusa del hígado, riñones y bazo. Áreas de infarto fueron observadas en ambos riñones, borde craneal del bazo y mesenterio (Figuras 2A, B, C y D).

Muestras de aorta fueron fijadas en formol neutro tamponado al $10 \%$ y enviadas al laboratorio de histopatología. Las muestras fueron procesadas mediante protocolos de rutina, se incluyeron en bloques de parafina, se realizaron cortes de $5 \mu \mathrm{m}$, y las secciones de tejido fueron colocados sobre portaobjetos de vidrio para coloración con hematoxilina y eosina. Los cortes fueron examinados mediante microscopía óptica. Los hallazgos microscópicos describen en la túnica adventicia, tejido adiposo y mesotelio adyacente de la aorta la presencia de un infiltrado leucocitario perivascular difuso y severo, constituido por neutrófilos, linfocitos y macrófagos. Hay focos de hemorragia y congestión vascular severa. En la túnica íntima se evidenció la pérdida de continuidad sin más lesiones significativas (Figura 3). El diagnóstico fue de arteritis, periarteritis y esteatitis aguda. Con base a las lesiones macroscópicas, se determinó que la causa de muerte del animal fue un choque hipovolémico por ruptura de aorta debido a migración de larvas de S. lupi.

\section{Discusión}

El shock hipovolémico por ruptura de aorta es una patología raramente diagnosticada en cánidos y su pronóstico en medicina veterinaria es grave debido a la pérdida masiva y rápida de volumen sanguíneo circulante. La muerte súbita, en algunos casos durante una rutina física, sería la presentación clínica más clásica de ruptura aguda de aorta, diferente a lo que puede ocurrir cuando se produce la disección de este vaso sanguíneo (Boulineau et al., 2005).

Diversas causas han sido asociadas a este evento, entre ellas, defectos de origen hereditario en los componentes del tejido conectivo, incluidas la elastina o las fibrillas de colágeno, similares a los que se presentan en el síndrome de Marfan en humanos (Lenz et al., 2015). Para confirmar el diagnóstico de este síndrome en un caso de ruptura aortica se requiere la ejecución de pruebas hereditarias que no fueron realizadas en el presente reporte. La migración de larvas de parásitos como el Angiostrongylus vasorum (Mozzer y Lima, 2012) y el S. lupi (Hamir, 1984; Rinas et al., 2009), han sido igualmente asociados a la disección y ruptura de aorta en perros domésticos o silvestres.

Las larvas de $S$. lupi causan necrosis, hemorragia y exudación de neutrófilos en la pared de los vasos en los que migran. Las lesiones producidas generalmente cicatrizan por completo; no obstante, el tejido elástico y muscular de la pared de la aorta torácica se vuelve fibrótico y, en ocasiones, se mineraliza para formar cicatrices permanentes en la túnica íntima y aneurismas en número y tamaños diferentes (van der Merwe et al., 2008). Las complicaciones de los aneurismas aórticos incluyen debilidad estructural de la aorta que puede conducir a la disección de la túnica media o a rotura fatal de la pared arterial (Pretre y Vonsesser, 1997).

Las lesiones histopatológicas descritas en el presente reporte (Figura 3), como un infiltrado leucocitario difuso y severo, constituido por neutrófilos, linfocitos y macrófagos en las túnicas media y adventicia, tejido adiposo perivascular y mesotelio adyacente de la aorta, sugieren un agente inflamatorio que lesionó el vaso, y como fue observado duran- 

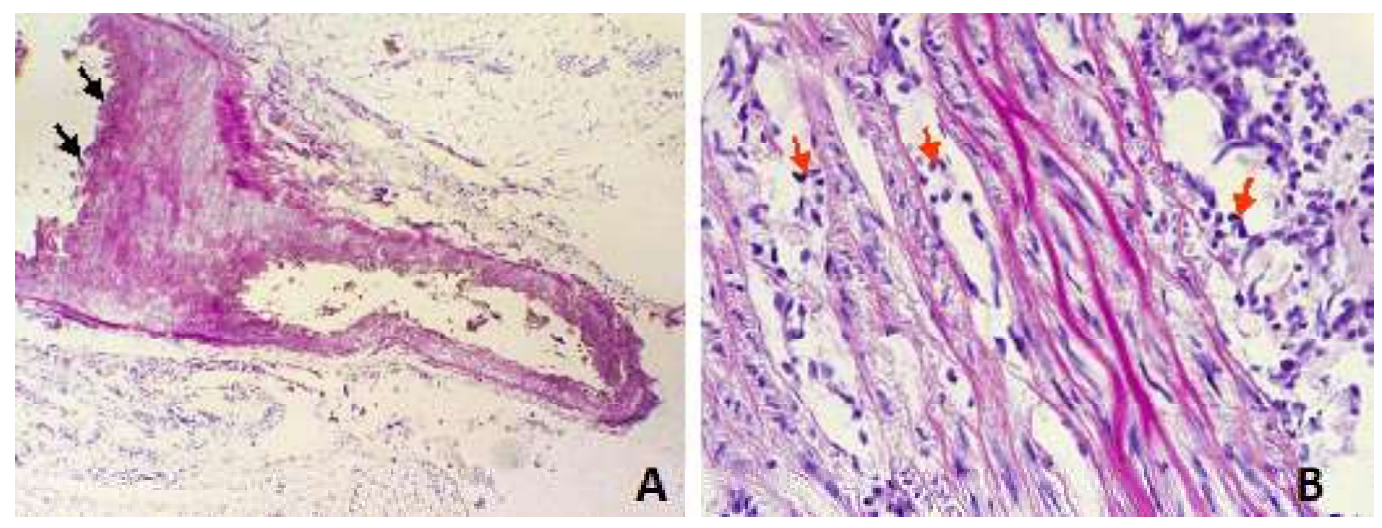

Figura 3. Alteraciones histológicas producidas por la migración de Spirocerca lupi. A. Lesión endotelial de la aorta con pérdida de continuidad (flechas negras), se observa ruta de migración del agente etiológico formando un canal en dirección a la adventicia del vaso (cabeza de flecha) (HE 40x). B. Túnica media de la aorta donde se observa infiltrado inflamatorio (flechas rojas) y desorganización de las fibras elásticas

te la realización de la necropsia, una larva de S. lupi estaría directamente comprometida en la ruptura del vaso. Se sugiere que la gravedad del daño puede estar asociada con la cantidad de larvas que migran; sin embargo, en este estudio solo una larva fue observada, lo que puede sugerir algún tipo de alteración en los componentes de la aorta del animal (van der Merwe et al., 2008).

Otras lesiones encontradas fueron la nefritis intersticial - perivasculitis no supurativa - microtrombosis, esplenitis congestiva aguda - hiperplasia linfoide, hiperplasia linfoide reactiva - hemorragia. Las lesiones isquémicas observadas en diversos órganos están asociadas a la formación de coágulos en el lugar de la ruptura del vaso y la migración de estos hasta órganos distantes a través de circulación sistémica, ocasionado de esta forma áreas de infarto. El tromboembolismo aórtico como consecuencia de $S$. lupi es un evento poco frecuente que rara vez ha sido documentado en la literatura (Gal et al., 2005). El hallazgo de un agente etiológico parasitario asociado a la ruptura de la pared aórtica no excluye la posibilidad de alteración heredable en los componentes de esta. Se requiere realizar otras técnicas de coloración en cortes histológicos de la aorta para verificar alteraciones en el tejido conjuntivo de la pared.

\section{Literatura Citada}

1. Bailey WS. 1963. Parasite and cancer: sarcoma in dogs associated with Spirocerca lupi. Ann Ny Acad Sci 108: 890-923. doi: 10.1111/j.17496632.1963.tb13429.x

2. Banning AP, Pillai R. 1997. Nonpenetrating cardiac and aortic trauma. Heart 78: 226-229.

3. Biasato I, Zanatta R, Maniscalco L, Evangelista R, Iotti B, Iussich S. 2018. Left subclavian artery dissection associated with connective tissue abnormalities resembling Marfan-like syndrome in an English bulldog. J Vet Cardiol 20: 136142. doi: 10.1016/j.jvc.2018.02.001

4. Boulineau TM, Andrews-Jones L, Van Alstine W. 2005. Spontaneous aortic dissecting hematoma in two dogs. J Vet Diagn Invest 17: 492-497. doi: 10.1177/ 104063870501700518 
5. Gal A, Kleinbart S, Aizenberg $Z$, Baneth G. 2005. Aortic thromboembolism associated with Spirocerca lupi infection. Vet Parasitol 130: 331-335. doi: 10.1016/j.vetpar.2005.03.037

6. Gutierrez G, Reines HD, and WulfGutierrez ME. 2004. Clinical review: haemorrhagic shock. Crit Care 8: 373381. doi: $10.1186 / \mathrm{cc} 2851$

7. Hamir AN. 1984. Perforation of the thoracic aorta in a dog associated with Spirocerca lupi infection. Aust Vet J 61: 64. doi: 10.1111/j.1751-0813.1984.tb07195.x

8. Joubert KE, McReynolds MJ, Strydom F. 2005. Acute aortic rupture in a dog with spirocercosis following the administration of medetomidine. J S Afr Vet Assoc 76:159-62. doi: 10.4102/ jsava.v76i3.418

9. Kirberger RM, Clift SJ, van Wilpe E, Dvir E. 2013. Spirocerca lupi associated vertebral changes: a radiologicpathologic study. Vet Parasitol 195: 8794. doi: 10.1016/j.vetpar.-2012.12.039

10. Lenz JA, Bach JF, Bell CM, Stepien RL. 2015. Aortic tear and dissection related to connective tissues abnormalities resembling Marfan syndrome in a Great Dane. J Vet Cardiol 17: 134-141. doi: 10.1016/j.jvc.2015.01.001
11. Mozzer LR, Lima WS. 2012. Rupture of the thoracic aorta associated with experimental Angiostrongylus vasorum infection in a dog. Parasite 19: 189-191. doi: 10.1051/parasite/2012192189

12. Pretre R, Von Segesser LK. 1997. Aortic dissection. Lancet 349: 14621464. doi: 10.1016/S0140-6736(96)09372-5

13. Ramachandran PV, Shakir SA, Ramakrishnan R. 1984. Spirocercosis in canines-a necropsy survey. TNJVAS 13: 132-135.

14. Rinas MA, Nesnek R, Kinsella JM, DeMatteo KE. 2009. Fatal aortic aneurysm and rupture in a neotropical bush dog (Speothos venaticus) caused by Spirocerca lupi. Vet Parasitol 164: 347-349. doi: 10.1016/j.vetpar.2009.05.006

15. van der Merwe LL, Kirberger RM, Clift S, Williams M, Keller N, Naidoo V. 2008. Spirocerca lupi infection in the dog: a review. Vet J 176: 294-309. doi: 10.1016/j.tvj1.2007.02.032

16. Wey AC, Atkins CE. 2000. Aortic dissection and congestive heart failure associated with systemic hypertension in a cat. J Vet Intern Med 14: 208e13. doi: 10.1111/j.1939-1676.2000.tb02239.x 